\title{
EL AZAROSO PASO DEL RUBICÓN DE LA RESTAURACIÓN MONUMENTAL EN ESPAÑA
}

\section{(THE DIFFICULT CROSSING OF THE RUBICON OF THE MONUMENT RESTORATION IN SPAIN)}

Lluís de Grassot, Cronista y Articulista de Arquitectura

Fecha de recepción: 4-VIII-93

\section{RESUMEN}

La disciplina de la restauración monumental vive en España un momento delicado después de atravesar uno de los periodos más complejos iniciados tras el proceso de recuperación de la normalidad democrática. Algunas circunstancias politicas, culturales $\gamma$ profesionales de la década de los 80 , hicieron que nuestros monumentos pasaran del olvido a un protagonismo no siempre sensato.

Hoy, superadas algunas facetas de aquella coyuntura, se recogen los frutos de sus aspectos positivos la recuperación de la "actitud creativa" frente al proyecto, la valoración del monumento como documento histórico, la afirmación de la necesaria colaboración interprofesional), pero continitan sufriéndose algunos vicios crónicos (las actitudes profesionales arrogantes, el inmovilismo de una parte de la Administración, la legislación obsoleta, la dificultad de aprendizaje).

El autor manifiesta su esperanza de que, en el contexto de una evidente "nueva mentalidad" respecto al patrimonio monumental, la crisis económica favorezca la consolidación de una "cultura de la conservación" paralela a la renovada e imprescindible cultura de la restauración.

\section{SUMMARY}

The monument restoration in Spain is passing through a delicate moment after going through one. of the most complex periods which started after the process of social recovery leading to democracy. Some political, cultural and professional circumstances of the decade of the 80s, made our monuments go from oblivion to playing a not always sensible leading role.

Today, when some facets of that situation have been overcome, the fritits of its positive aspects can be collected (the retrieval of the "creative attitude" towards the project, the appreciation of the monument as historical document, the acceptance of the necessary interprofesional collaboration). Nevertheless, some chronic vices are still being suffered from (the arrogant attitudes of the professionals, the low efficiency level of one part of the Administration, the obsolete legislation, the difficulties in trainning).

The author expresses his hope that, in the context of an obvious "new mentality" towards our heritage of monuments, the economic crisis will favour the consolidation of a "conservation culture" parallel to the renewed and indispensable culture of restoration.
La restauración monumental parece condenada a desarrollarse entre permanentes tensiones y polémicas, debido quizá a la complejidad congénita tanto de sus fines como de sus medios. En España se vive ahora un momento delicado después de un período -la década de los 80 - especialmente confuso debido a una coyuntura relacionada con la entonces reciente recuperación de la normalidad democrática.

De las circunstancias que favorecieron aquella confusión yo señalaría como más significativas, en primer lugar -y utilizando palabras del historiador Javier Rivera-, la "fiebre restauradora, intervencionista y rehabilitadora" que se desató a partir del inicio de la década. Una fiebre que tuvo dos causas fundamentales: una, la crisis económica que revalorizó el viejo parque edificado, incluido el patrimonio monumental, que incrementaba así su valor de uso. Y, en segundo lugar, la cantidad de monumentos infrautilizados y de déficits de equipamientos heredados de la dictadura, que sugirió a las nuevas administraciones democráticas -especialmente las regionales y 
locales- el volver los ojos hacia ese patrimonio para tratar de solventar dos problemas a la vez.

La revalorización económica del patrimonio dejó algunas secuelas negativas como consecuencia de los usos y tratamientos inadecuados que sufrieron muchos elementos por causa de ese nuevo interés más económico que cultural. En contrapartida, muchos de ellos -bien tratados o no- deben su supervivencia a la crisis. El repentino afecto de la Administración hacia el patrimonio, sin embargo, produjo más perjuicios que beneficios. Según el profesor Rivera, las nuevas instituciones democráticas españolas, creyendo "monumentalizarse a sí mismas al ocupar monumentos", negando "su propia significación de instituciones nuevas y jóvenes", lo que hicieron fue "destruir o alterar los monumentos... dentro de la más preclara concepción de la arquitectura histórica como instrumento institucional del poder" (1).

A mi juicio, como causa de la parcela de "fiebre" que correspondió a los poderes públicos habría que admitir también, en algunos casos, la asunción honesta de las reivindicaciones populares y profesionales que se dieron en muchas de nuestras ciudades en los últimos años de la dictadura. Además, aunque no muy numerosos, encontraríamos ejemplos de conductas correctas por parte de la Administración. En general, sin embargo, el comentario del profesor Rivera tiene fundamento: la reutilización del patrimonio en manos de los nuevos administradores tuvo más sombras que luces. Aún son palpables en muchos de nuestros monumentos los efectos de la supeditación de las actuaciones a objetivos de prestigio o a usos decididos sin suficiente reflexión, o de los aturullamientos producidos por las prisas electorales.

\section{DESBARAJUSTE PROFESIONAL}

La segunda circunstancia que favoreció la confusión -y sigo en esto al arquitecto Antoni González ${ }^{(2)}-$. guarda relación con los profesionales encargados de instrumentar la masiva intervención en nuestro patrimonio. Cuando se dio el debate sobre restauración monumental más rico de los producidos a lo largo de nuestro siglo (el coincidente con la reconstrucción europea posterior a la Segunda Guerra Mundial), nuestra cultura sesteaba bajo los efectos de una dramática resaca. Y, desgraciadamente, el debate no se produjo tampoco en España en los años del desarrollismo, alejados como estaban entonces de estas materias tanto los arquitectos de la cultura (imbuidos aún por la infravaloración que el

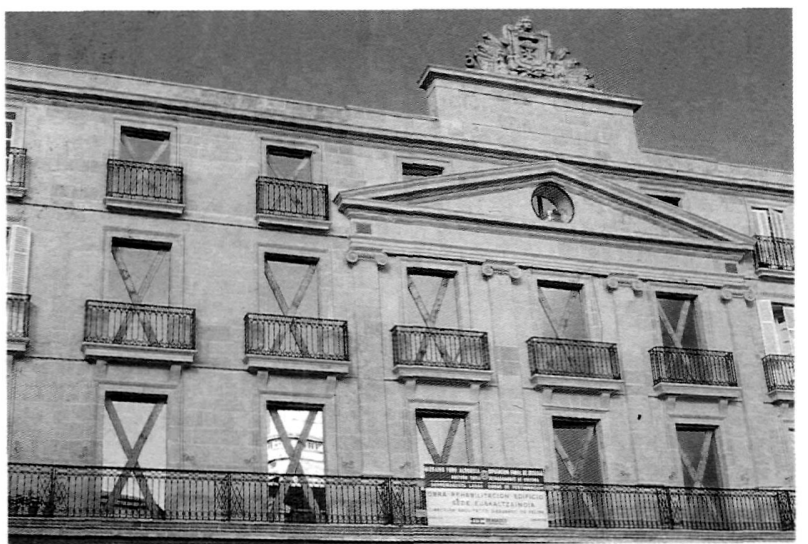

El "fachadismo". un estigma de nuestros centros bistóricos.

Movimiento Moderno había hecho de la historia), como los historiadores ensimismados en su retiro universitario.

Por ello, cuando al final de los años 70 apareció la fiebre, los profesionales españoles acusaron el ayuno de teoría que padecían. Ayuno que se mantendría durante la década siguiente, ya que el frenesí de la propia fiebre aconsejó aplazar de nuevo el debate. (En nuestro país, paradójicamente, la confusión conceptual de esos años se dio por falta, no por exceso -como en el caso de Italia- de formulaciones teóricas).

La ausencia de preparación y de reflexión, sin embargo, no arredró a muchos arquitectos a incorporarse a la práctica restauratoria. La mayor parte de ellos accedían por primera vez al mercado de trabajo público y no estaban dispuestos a renunciar a él así como así, sólo porque las circunstancias condujeran gran parte de ese trabajo hacia el esotérico campo, hasta entonces desprestigiado, de la intervención en los monumentos. Fue entonces cuando se formuló en el seno del gremio la capciosa idea de que "para restaurar bastaba con ser un buen arquitecto" (si se era capaz de diseñar torres blancas, cómo no se iba a saber restaurar catedrales góticas...).

El arraigo en la nueva Administración del hábito de seleccionar proyectistas en función de su prestigio en otros campos de la profesión o del eco publicitario del encargo; la falta de mecanismos de control de la joven Administración frente a la arbitrariedad o la torpeza de los profesionales; la ineficacia de una ley de patrimonio (aprobada en la mitad de la década) concebida para frenar a los restauradores de la vieja escuela más que para prevenir los males de una fiebre aún no anunciada cuando la ley fue redactada, y el silencio cómplice de una autista comunidad científica y universitaria (que a lo sumo se manifestó para 


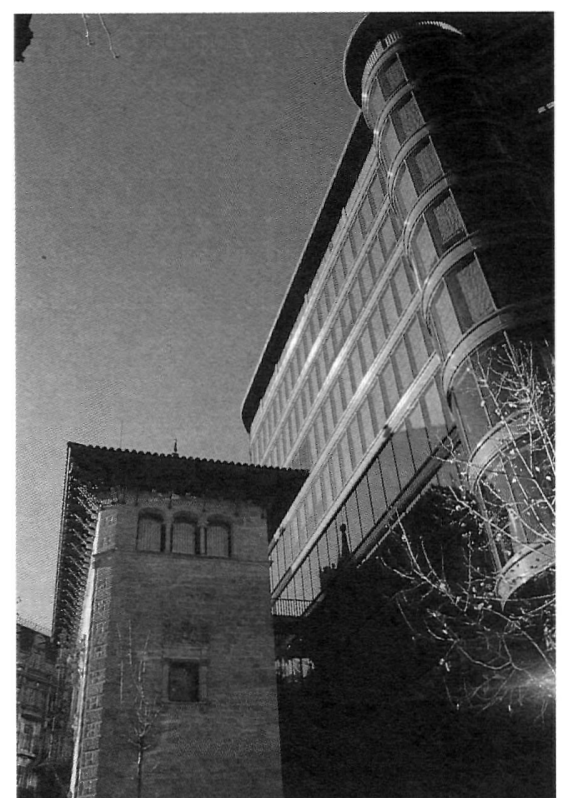

"Sodomía arquitectónica" en Barcelona. Casa Serra de Puig i Cadafalch en la Diagonal.

anatematizar, pero sin dar alternativas) facilitaron el desbarajuste profesional.

También atizaron la confusión los intentos de presentar la restauración como un puro ejercicio tecnológico, reservado a expertos especialistas, o una técnica fácil de asumir por animosos diletantes desde las escuelas-taller o por técnicos diestros en "rehabilitar" el parque de viviendas; actitudes casi siempre derivadas de haberse trasladado al próspero campo de la restauración viejas o nuevas disputas competenciales entre colectivos profesionales.

Los efectos negativos de estas circunstancias fueron inevitables. En algunos deteriorados monumentos el escaso presupuesto público disponible se malbarató en incontroladas e innecesarias obras "de diseño". (En este sentido, la obra del arquitecto Javier Bellosillo en el monasterio riojano de Santa María de Nájera -denunciada incluso, aunque tarde, por los sorprendidos historiadores ${ }^{(3)}$ - quedará para siempre como paradigma). En otros casos, la prisa y la ignorancia provocaron daños irreparables. Sirva de ejemplo la actuación en los edificios góticos de la calle de Montcada de Barcelona para instalar el museo Picasso, obra en la que la indiscutible calidad del diseño de Jordi Garcés y Enric Sòria no justifica la falta de método restaurador.

Tampoco se salvaron del desbarajuste los conjuntos urbanos y los centros históricos, en los que se enquistaron conductas bien diversas: desde la versión actualizada de la ramplonería historicista (bien saben de eso Sevilla, Toledo y tantos otros conjuntos históricos) hasta el fachadismo - la exclusiva valoración anecdótica de las fachadas costosamente "conservadas" mientras el edificio al que pertenecieron se hacía desaparecer (hay ejemplos en todas nuestras ciudades)-, la sodomización de la arquitectura -la agresión de las traseras de un edificio histórico por otro "moderno" (como el caso de Can Serra en Barcelona)-, o la concepción de la restauración como simple cosmética, como en la misma Barcelona, que se puso guapa a costa de embadurnar su arquitectura histórica.

\section{ASPECTOS POSITIVOS DE UNA DÉCADA}

Pese a todo, esta agitada década de la restauración española tuvo aspectos sólidamente positivos. En primer lugar, el hecho de que la sociedad aceptara que su patrimonio podía asumir un legítimo papel simbólico y, por tanto, fuera conveniente su conservación activa. Es sintomático que los dos acontecimientos universales del 92 -la Expo y los Juegos Olímpicos- tuvieran sus sedes más representativas en edificios históricos (la cartuja de Santa María de las Cuevas y el Estadio Olímpico), restaurados y recuperados para ello. Fue un hecho especialmente significativo en el caso de Barcelona, ya que conservar la desvencijada fachada de aquel viejo estadio simboliza la firme voluntad de no renunciar al pasado en el momento de proyectar el futuro.

En segundo lugar, cabe considerar como aspecto positivo desde una óptica profesional la recuperación de una actitud tradicional -casi un "invariante castizo"- de la intervención en el patrimonio español: la actitud creativa frente al proyecto de intervención. Los arquitectos españoles, en "clara contestación a las posturas historicistas que habían llenado los últimos decenios de realizaciones sobre los

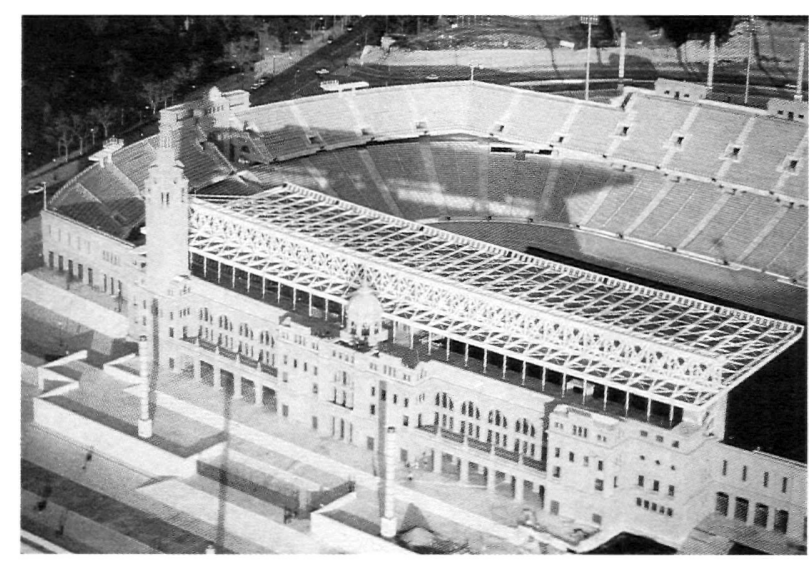

Estadio Olimpico de Barcelona; la voluntad de no renunciar al pasado en el momento de proyectar el futuro. 


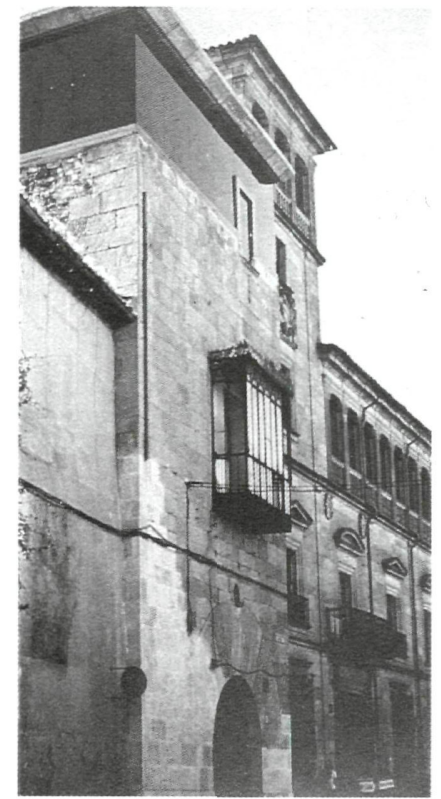

El desafiante manifiesto del arquitecto Pulin en Salamanca.

monumentos" -señaló Alfonso Muñoz Cosme en su lúcido análisis de aquellos años-, adoptaron "una forma de intervenir sobre los edificios en la que el nuevo diseño adquiere un papel protagonista en la actividad proyectiva".(4)

Esta actitud creativa (más relacionada con la herencia cultural que con la adhesión a las nuevas teorías -la "restauración crítica" de Renato Bonelli era casi desconocida aquí (5)-) llegaría a constituir un signo de identidad de la restauración española en el exterior. La atractiva y desafiante actuación del arquitecto Pulín es una torre de Salamanca, censurada poco después, fue su más atrevido manifiesto.

También son ejemplos válidos de esa aportación creativa a nuestros monumentos -por citar sólo aquellas obras cuya valoración han destacado los críticos de este período ${ }^{(6)}$ - la adaptación de los bajos de la Casa Thomas de Barcelona por el estudio PER, el pabellón de la Merced del Hospital de San Pablo de Barcelona y la torre de la Manresana, de Víctor Argentí y Antoni González, la reforma del convento de San Benito de Alcántara, de Miguel de Oriol y Dionisio Hernández-Gil, la intervención de Luis Burillo y Jaime Lorenzo en la iglesia de San Juan de Daroca, las primeras obras del renovado Servicio de Catalogación y Conservación de Monumentos de la Diputación de Barcelona (Santa Càndia d'Orpí, Sant Vicenç de Malla, el acueducto de Sant Pere de Riudebitlles, proyectadas por Antoni González), la intervención en San Hipólito de Támara (Mas Guindal, Clemente, Cases y de la Quintana, arquitec-

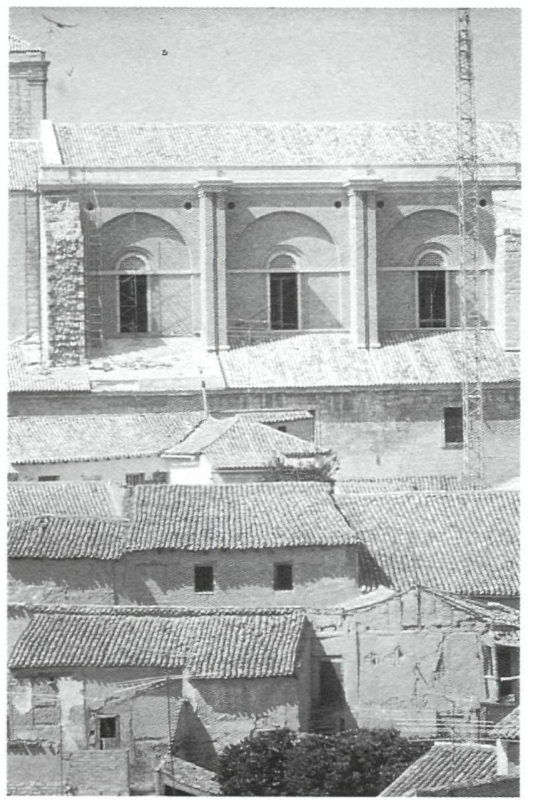

Reconstrucción de la iglesia de la Santa Cruz. Medina de Rioseco.

tos), la reconstrucción de la iglesia de la Santa Cruz de Medina de Rioseco por José Ignacio Linazasoro (discutible. quizá en cuanto a sus aspectos programáticos), o la actuación de Enrique Nuere y Pío García Escudero en el convento de Santa Clara de Salamanca. Incluso algunas adaptaciones de edificios históricos como sedes de parlamentos autonómicos -quizá la más alta manifestación de la "arquitectura histórica como instrumento institucional del poder"- se hicieron con buen tino, como en Santander, Fuensaldaña, Logroño, Zaragoza y Sevilla.

Son obras que compartieron los aspectos positivos de la praxis restauradora de la década con otras, no menos dignas, de pura conservación, como la de Antonio Fernández Alba en la real Clerecía de San Marcos de Salamanca, "una intervención de carácter estructural en la que [el autor] se autorrestringía a

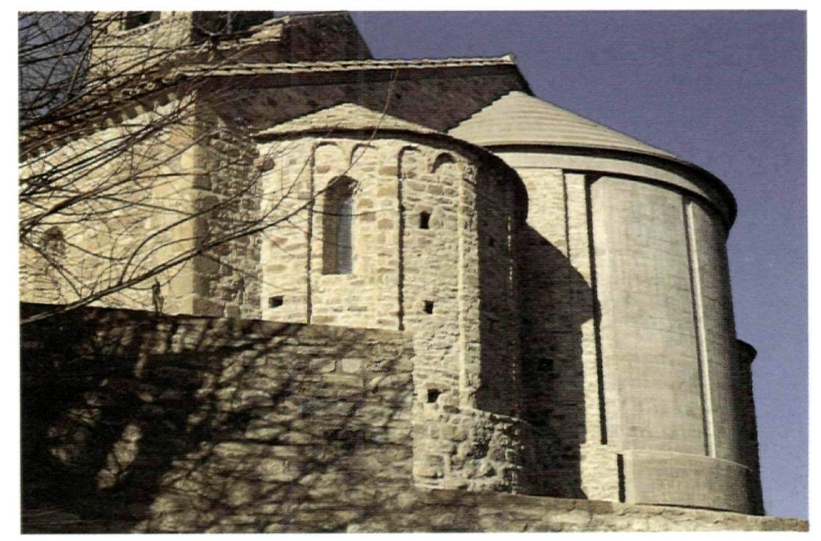

Nueva cabecera de la iglesia de San Vicenç de Malla (Barcelona). 
aplicar el verdadero debate de la moderna restauración crítica" ${ }^{(7)}$, o de restauración estricta -así se tendrían que calificar algunas excelentes obras de Amparo Berlinches, San Martín de Valdilecha, por ejemplo, o la actuación en la catedral de Sevilla, dirigida por Alfonso Jiménez y Pedro Rodríguez-, o de "desrestauración", como define Javier Rivera ${ }^{(8)}$ la actuación de Antoni González en la iglesia de Sant Cugat dels Gavadons de Collsuspina.

La línea divisoria, en ocasiones ciertamente sutil, entre estas obras y los dislates que por desgracia conforman el panorama general de aquella época, tendría que situarse en el tratamiento de los valores documentales del monumento en el que se intervenía. Porque el caos no se produjo -como pretenden algunos tratadistas- por la propia actitud creativa, válida en sí misma, sino porque demasiado a menudo iba acompañada del olvido de ese valor docu- mental y las actuaciones devenían destructivas o desnaturalizadoras del monumento.

El arquitecto andaluz Alfonso Jiménez, uno de los pioneros en teorizar esta actitud creativa ("la restauración... es una actividad artística", dijo, "que exige el desarrollo de todas las cualidades del arquitecto, sin renunciar a su propia capacidad creativa... ") ya advirtió que esa conducta del arquitecto se debe entender como "una actuación subjetiva pero no arbitraria". (9)

La re-significación de la arquitectura histórica, "bien se materialice por mecanismos creativos, bien por mecanismos de recuperación mimética", dice Antoni González "supone a menudo la alteración del monumento, una alteración -pocas veces reversible, aunque se afirme lo contrario- que puede poner en peligro... el valor documental (informativo y conme-

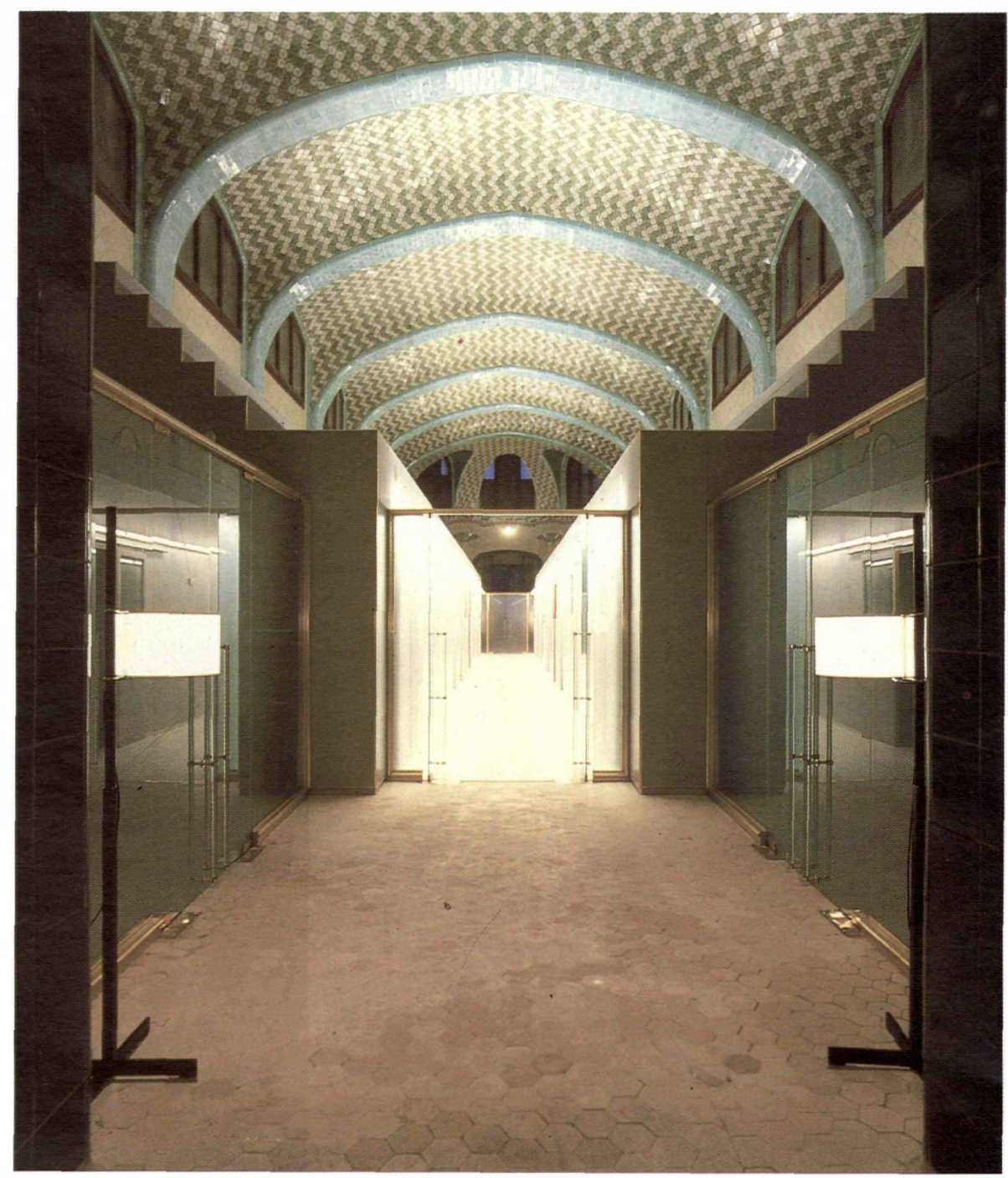

Área obstétrica del Hospital de San Pablo de Barcelona. 


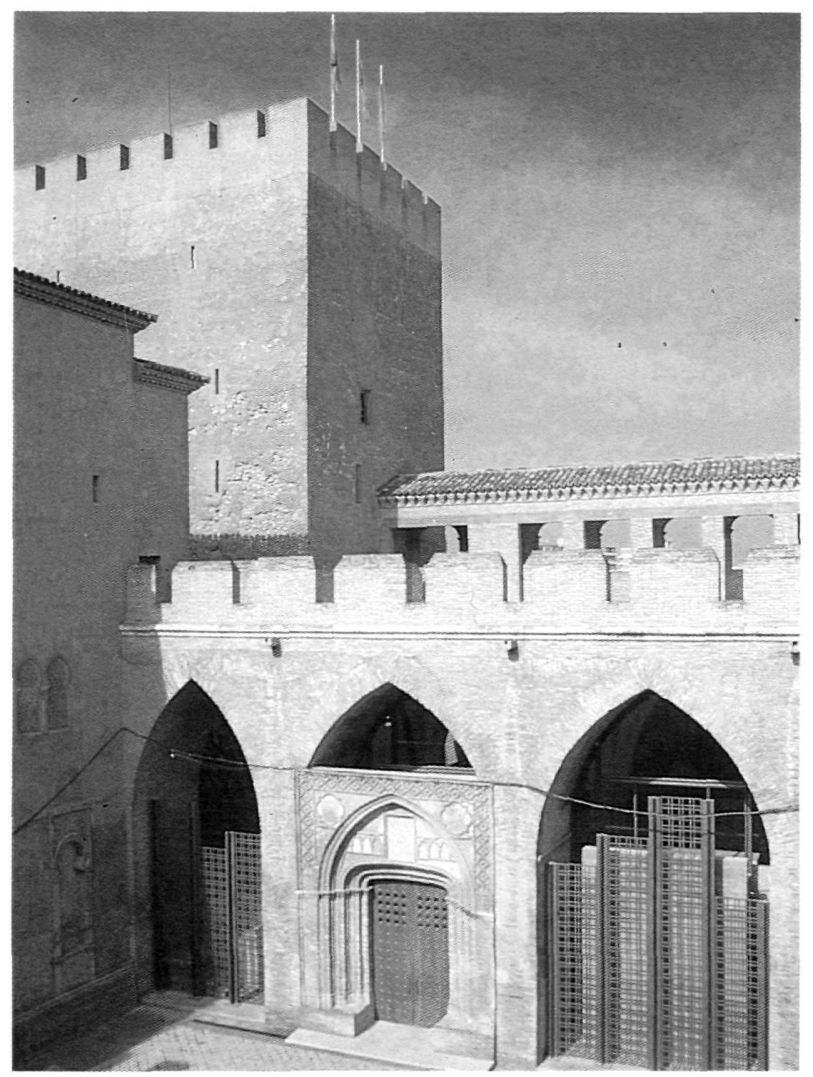

Las Cortes de Aragón en el Palacio de la Aljafería de Zaragoza.

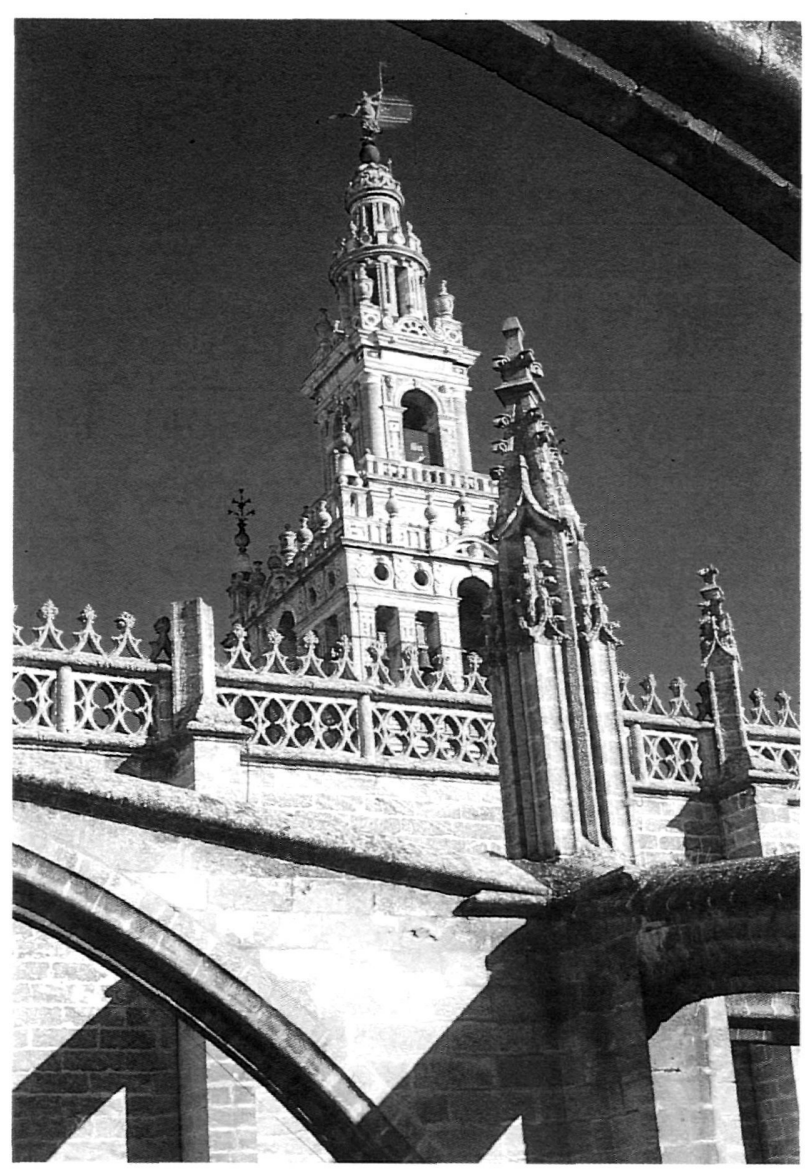

La catedral de Sevilla presidida por la Giralda. morativo) del monumento, un valor que se ha de tener presente siempre al plantear estrategias y proyectos de intervención en el monumento... ya que puede poner límites insuperables a su transformación". (10) El olvido de estas consideraciones, aparentemente elementales, fue la causa del caos.

Pero como ocurre habitualmente, del propio mal surgieron el antídoto y la vacuna. Las reacciones -desde muy diversas ópticas y mentalidades, pero casi siempre con el denominador común de la exigencia de más respeto por los aspectos documentales y científicos del monumento- no se hicieron esperar. Ese sería el segundo gran aspecto positivo de la década prodigiosa de nuestra restauración.

\section{REACCIÓN FRENTE AL CAOS}

La reacción más contundente provino de los profesionales de la historia (especialmente de la arqueología, tan dados a endosar a los demás las causas de sus males) y de diversas técnicas y ciencias relacionadas con la restauración. Más que por reclamar respeto hacia el monumento como documento, empezaron por exigir el compartimiento de los beneficios profesionales de la fiebre restauradora, hastiados de que en esto de la restauración siempre hubieran mandado los mismos. Trataron de hacerlo, sin embargo, por un camino erróneo: negar el carácter arquitectónico de la restauración monumental y preconizar un conservacionismo radical.

Los cientos, quizá miles, de simposios, congresos, másters, jornadas y cursos convocados en España en aquellos años al amparo de la fiebre, con una presencia cada día más abundante de esos profesionales, fueron testigos de sus tensiones con los arquitectos. Pero pronto las reivindicaciones darían frutos positivos, una vez que se supieron encauzar hacia la creación de las bases de una colaboración interdisciplinaria necesaria para mejorar el método de la restauración monumental, lo que tendría que permitir la superación tanto del inmovilismo como de la arbitrariedad.

En esta tarea tuvieron un papel importante algunos servicios de la Administración pública, especialmente el más antiguo, el de la Diputación de Barcelona, que preconiza una "restauración objetiva" basada en la consideración conjunta de los valores documentales, significativos y arquitectónicos del monumento y en un método exento de dogmatismos conceptuales, basado en el diálogo interdisciplinario. "11) En la defi- 
nición de esta "tercera vía equilibradora entre el conservacionismo a ultranza y la actuación crítica" fue decisiva - a juicio de Javier Rivera- la aportación del Servei catalán. ${ }^{(12)}$

Como consecuencia de esas reacciones se ha producido un cambio de mentalidad en cuanto a la actuación en los monumentos que ha empezado a ser patente en los primeros años 90 . Se ha avivado el debate conceptual, se ha producido el reencuentro con la cultura de la restauración de la primera mitad del siglo, se han consolidado las bases del diálogo y el trabajo pluridisciplinario, y se han equilibrado los aspectos técnicos, científicos y creativos de la restauración. (En este sentido debe considerarse como un síntoma el éxito de convocatoraria del simposio organizado por el Instituto Torroja sobre investigación científica y patrimonio ${ }^{(13)}$ y, en general, el auge de los simposios de restauración frente a loa cursos o cursillos de intervención, tan característicos de la década de los ochenta).

Algunas obras recientes -muy diferentes entre sí en los aspectos proyectuales, pero homogéneas en cuanto al método interdisciplinar que no excluye las aportaciones creativas cuando son convenientesson un buen reflejo de esta nueva mentalidad: las restauraciones de las iglesias de Santa Lucía del Trampal en Alcuéscar, Cáceres, (A. Almagro, L. Cámara, P. Latorre), Sant Quirze de Muntanyola y Sant Quirze de Pedret, Barcelona, (A. González), la Magdalena de Sevilla (A. Jiménez, F. Pinto, P. Rodríguez), y Santa María de Wamba, Valladolid, (J.L. de la Quintana, J.R. Boned), la restauración de la puerta de los apóstoles de la catedral de Valencia (J. Esteban), de la torre del Salvador de Teruel (A. Pérez, J.M. Sanz), del monasterio de Gradefes, León, (S. Mora), de la torre de Hércules en La Coruña (L. Cámara, P. Latorre), del Palau Güell de Barcelona

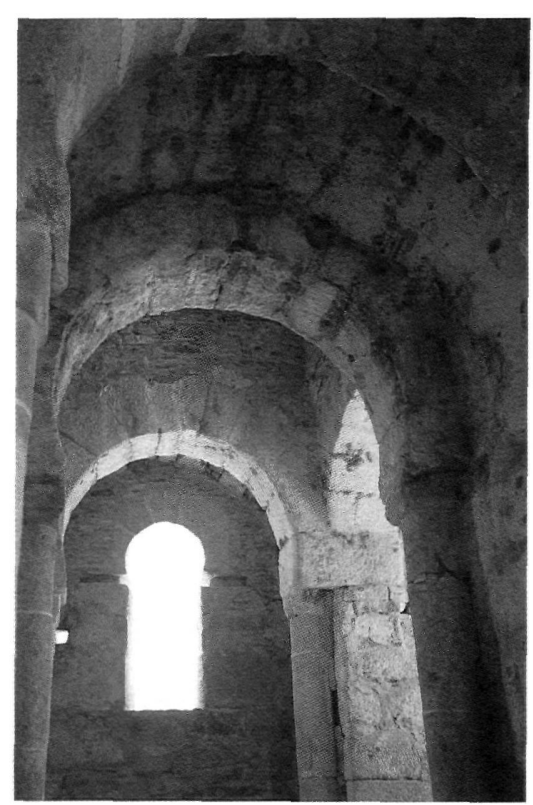

Interior de la iglesia visigoda de Santa Maria del Trampal.

(A. González, P. Carbó), de las fachadas de la carrera del Darro en Granada (I. Gárate) o la reconstrucción tipológica del teatro romano de Itálica en Santiponce, Sevilla, (A. Jiménez, F. Montero, P. Rodríguez).

\section{UN PERÍODO DELICADO}

Esta regeneración tanto en la teoría como en la praxis podía hacernos creer que, por fin, la disciplina había superado el caos de los años ochenta. Pero lo cierto es que el momento actual es muy delicado.

La sensibilidad colectiva ante la amenaza del patrimonio por causa de desaguisados urbanísticos está suficientemente arraigada en nuestra sociedad, que se curtió en estos sufrimientos durante la dictadura. (Las reacciones frente a los planes del MOPU/MOPT

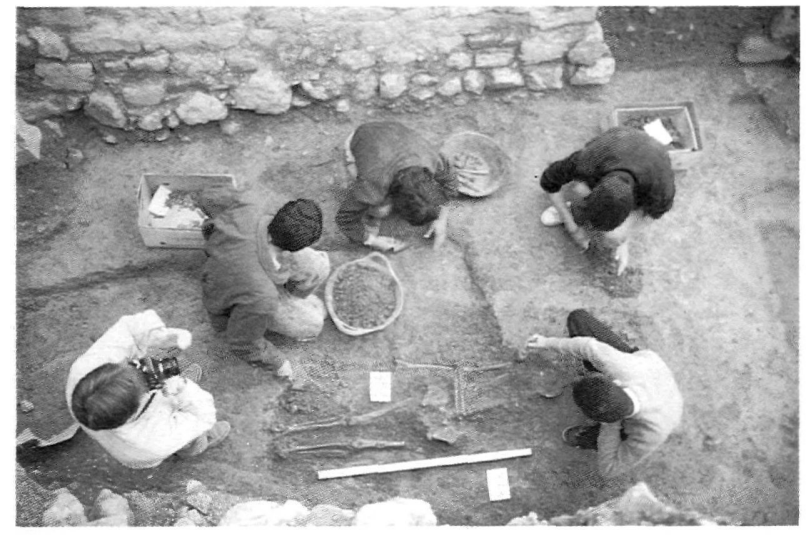

La valoración del monumento como documento histórico.

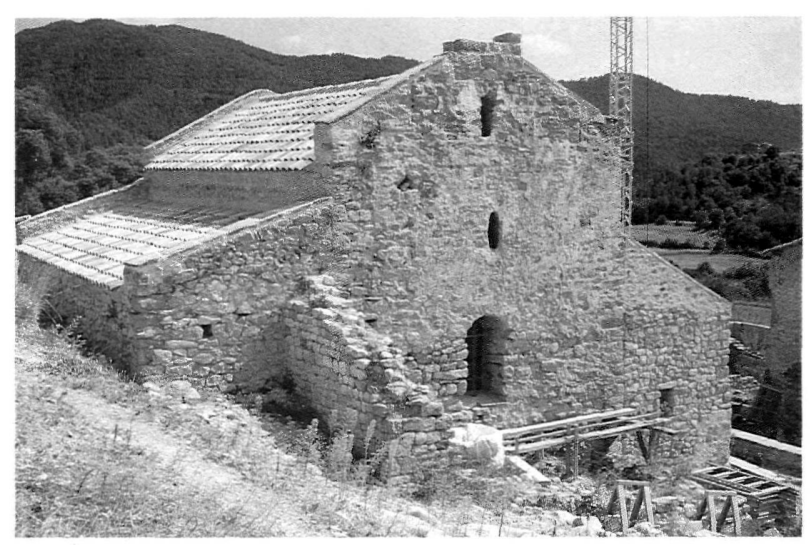

Iglesia prerrománica de Sant Quirze de Pedret (Barcelona). 


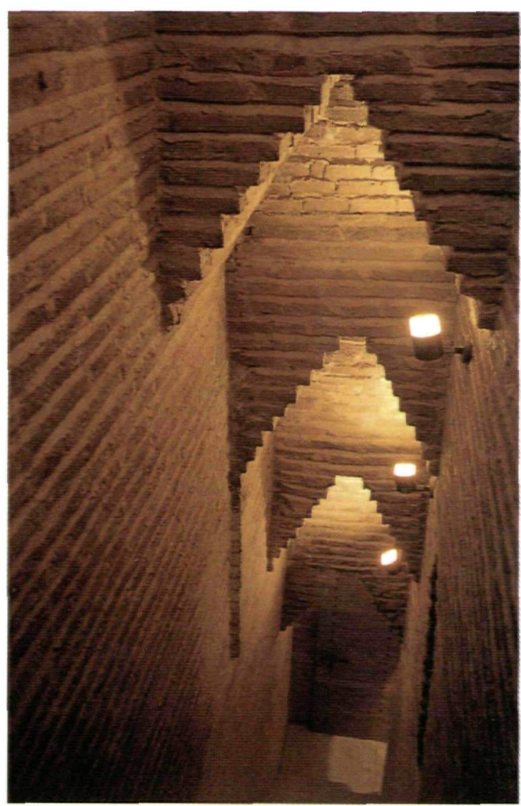

Interior de la torre del Salvador de Teruel.

en Soria -o, más recientemente, en Ávila- son buena muestra de ello). Pero la sensibilidad frente a los criterios de intervención no estaba hasta ahora tan a flor de piel. Las polémicas vividas los pasados años en los ámbitos profesionales han saltado ya a la opinión pública. La discusión sobre la legitimidad de la obra de los arquitectos Giorgio Grassi y Manuel Portaceli en el teatro romano de Sagunto, o sobre la eficacia social de la actuación de Salvador Pérez Arroyo y Susana Mora en el, ya deteriorado otra vez, monasterio de Carracedo (León) - dos obras de gran interés conceptual, por otra parte-, o sobre el difícil equilibrio entre el uso sin cortapisas de la ciudad y la conservación de los grandes monumentos urbanos o los restos arqueológicos (de máxima actualidad ahora en Segovia y Tarragona, por ejemplo), no ha hecho más que empezar, y aún puede tener consecuencias importantes en actuaciones futuras si el posicionamiento colectivo se decanta en uno u otro sentido, y pone en crisis criterios que ya parecían respaldados socialmente.

Otro aspecto intranquilizador del momento presente es que la Administración no avance, como podíamos esperar, ni en racionalizar las actuaciones y la distribución de recursos, ni en estructurar sus propios organismos. La legislación específica (tanto estatal como regional donde la hay) -uno de los mecanismos posibles para esa racionalización- sigue siendo ineficaz. Se persiste en el error de regular los criterios y se olvida establecer y exigir los métodos; laguna grave, como pudo comprobarse en la reciente sentencia judicial sobre la obra de Sagunto. (Nunca una norma legal había llegado con tanta rapidez a la obsolescencia como la ley española de 1985).

En cuanto a las inversiones, nuestros políticos siguen prefiriendo el beneficio inmediato de grandilocuentes y costosas obras estelares, o de la organización de gravosas exposiciones para difundir nuestro patrimonio, que conservar bien el mayor número posible de monumentos. Incluso los más significativos (a veces, paradójicamente, escenarios de esas magnas exposiciones) no son suficientemente atendidos. Es el caso de la Alhambra granadina, que cada día echa más en falta a don Leopoldo Torres Balbás.

Los servicios especializados, por su parte, no son dotados como debieran (ni en recursos humanos ni en utillaje) -salvo en honrosas excepciones de alguna Administración local o regional-, y se sigue dejando en manos de arquitectos contratados (muchas veces transeuntes de la disciplina) todo el peso de las actuaciones. Eso cuando los servicios no son desmantelados (como en el caso lamentable del Ayuntamiento de Barcelona, en otras épocas pionero de la Administración municipal en estos menesteres). Mientras, el Instituto de Conservación y Restauración de Bienes Culturales del ministerio de Cultura, no acaba de encontrar su lugar (imprescindible aún) en el nuevo Estado de las autonomías.

Desde el punto de vista metodológico también hay motivo para la zozobra. Es cierto que una buena cantidad de arquitectos que en estos últimos años tocaron de oído en la restauración han arrojado la toalla, y que la mayor parte de los que permanecen en la disciplina se esfuerzan en profundizar en la reflexión, el conocimiento especializado y el diálogo interprofesional. Pero la gestión que se hace desde la Administración favorece habitualmente que esa colaboración interdisciplinaria sea una falsedad (lle-

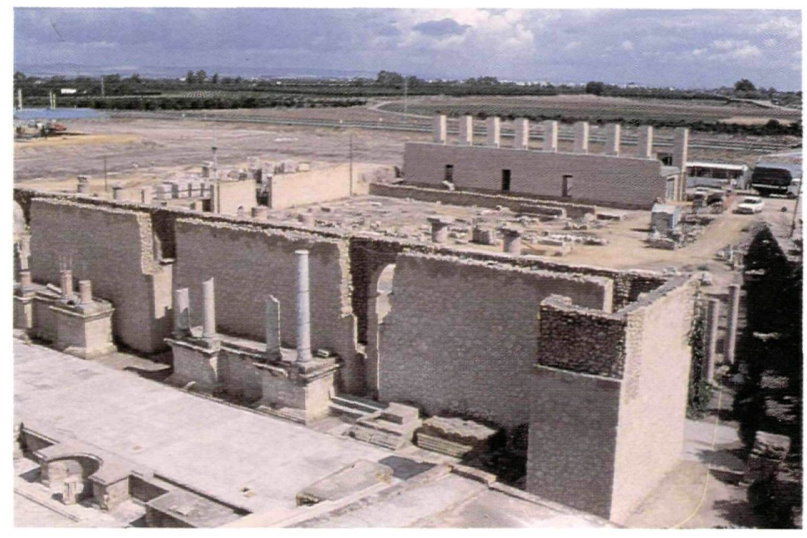

Reconstrucción tipológica del teatro romano de Itálica (Sevilla). 
vando siempre las de perder, justo es reconocerlo, los profesionales de la historia). Por otra parte, el desarrollo de las modernas técnicas (analíticas y terapéuticas) aplicables a la restauración, que ponen en manos de los técnicos unos recursos desproporcionados, pueden llegar a subvertir definitivamente la relación entre los fines y los medios.

En cuanto a las actitudes profesionales también queda un largo camino por recorrer. En primer lugar es constatable que, a pesar de la insistente reivindicación desde dentro de la restauración de su pertenencia a la disciplina de la arquitectura, en las tribunas donde se debate ésta sólo se presta atención a la actuación en los monumentos en función del valor, no del tratamiento del monumento en sí mismo, sino del diseño que dicho tratamiento haya exigido. (Una magnífica restauración que no requiera aportación creativa nunca será comentada; un disparate conceptual bien diseñado, sí). Para comprobarlo basta repasar la selección de obras de la II Bienal de arquitectura española ${ }^{(14)}$ o el anuario de 1993, publicado por Luis Fernández Galiano. ${ }^{(15)}$

Así, no es de extrañar que persistan actitudes colectivas estereotipadas que no favorecen el enriquecimiento conceptual ni el diálogo entre profesionales.
La reciente reacción visceral y corporativista de un destacado grupo de arquitectos en el caso del teatro romano de Sagunto - una obra en la que se evidencia la necesidad de una metodología rigurosa cuando se acomete la restauración con una actitud creativa- frustró (con ese ingenuo aforismo "es bueno todo lo que hacen los buenos") lo que pudo ser un diálogo sereno y reflexivo.

Tampoco en el ámbito de los historiadores y científicos se han serenado los ánimos, y abunda aún el estéril recelo hacia los profesionales de la arquitectura. (El propio caso de Sagunto ha sido una buena prueba. Qué pocas opiniones contrarias al proyecto se han basado en algo más que una descalificación genérica de la creatividad de los arquitectos). Actitudes positivas como la del historiador Javier Rivera "... nosotros como historiadores ... debemos admitir que se restaura para el presente e, incluso, para un inmediato futuro, aceptando en correspondencia los factores que condicionan espacial y temporalmente cada restauración ..." ${ }^{(16)}$, o las de la historiadora del arte Raquel Lacuesta y el arqueólogo Alberto López Mullor (plasmadas en una rica bibliografía sobre el trabajo interdisciplinario), son aún identificables y destacables precisamente por ser minoritarias.

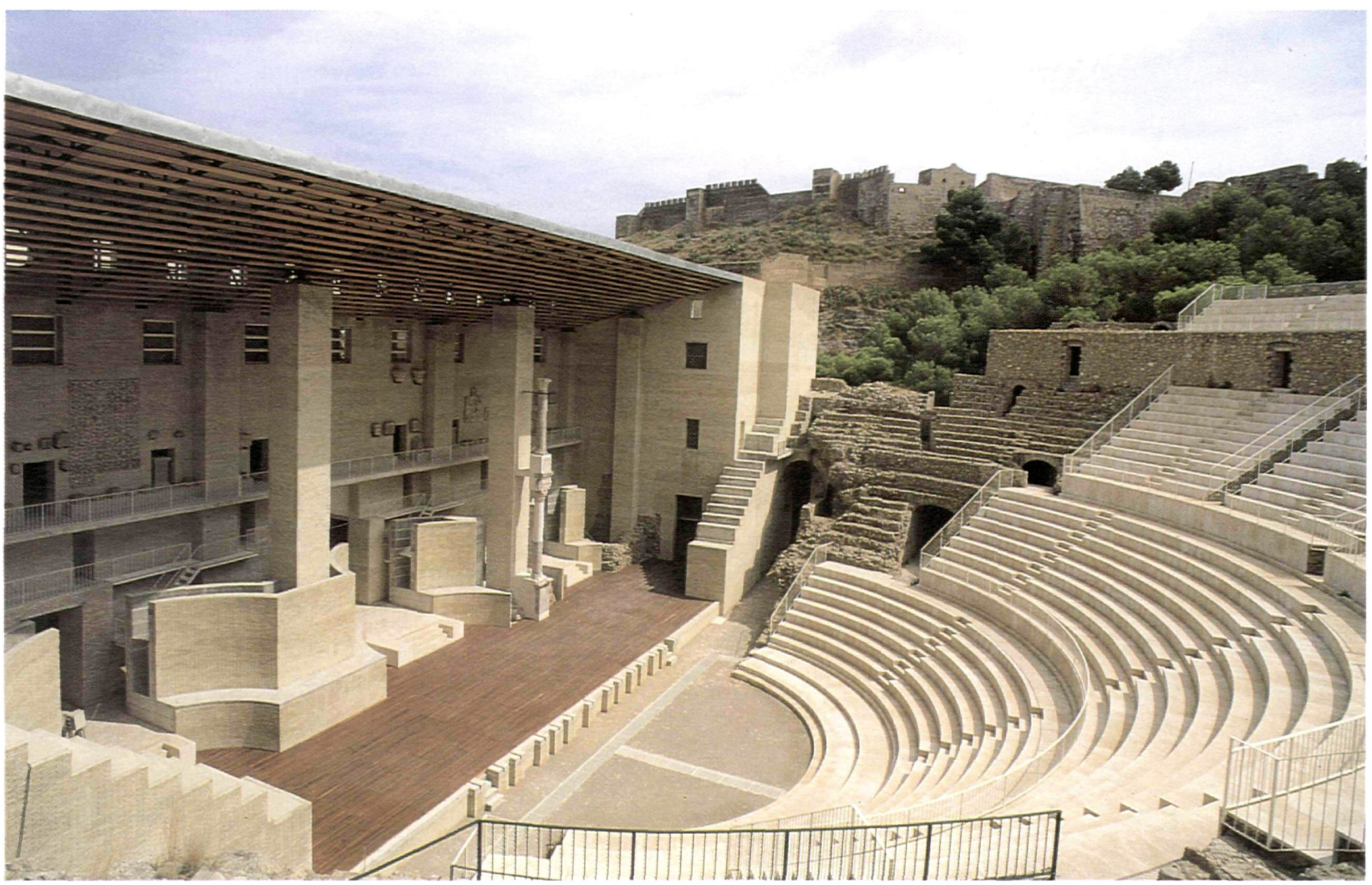

El nuevo teatro de Sagunto (Valencia). Irolo: . Hontserrat Baldomà. 
14

Informes de la Construcción, Vol. 45 n² 427, septiembre/octubre 1993

Quizá, en esta faceta profesional, sea un buen dato para la esperanza la creación en noviembre de 1992 , en el Palau Güell de Barcelona, de la Academia del Partal, una "asociación libre de profesionales de la restauración monumental", de ámbito estatal espanool, que ha nacido para favorecer ese diálogo interprofesional. Su silencio ante los acontecimientos de los últimos meses (especialmente ante la polémica desatada por la obra de Sagunto) no nos permite aún juzgar, sin embargo, el papel que esta academia pueda jugar en la regeneración de la restauración monumental española.

La dificultad del aprendizaje de la restauración es otro de los graves problemas abiertos en este delicado momento. El laberinto de másters y cursos al alcance de los posgraduados es francamente decepcionante, y en cuanto a los estudios de primer y segundo ciclo, en nuestras facultades y escuelas el panorama no es mejor. Las pocas nociones que se imparten son sesgadas, limitadas por la ausencia de un profesorado realmente preparado en la metodología de la restauración.

En las escuelas de arquitectura el problema es aún más grave, por incardinarse en un contexto de infravaloración de los aspectos constructivos de la arquitectura, y más aún de la arquitectura histórica. Así lo ha denunciado recientemene el arquitecto y catedrático José Luis González, que cree que en las escuelas españolas se reproduce todavía "el conflicto entre arte y ciencia, o sentimiento y razón" ${ }^{(1-)}$. "La creación

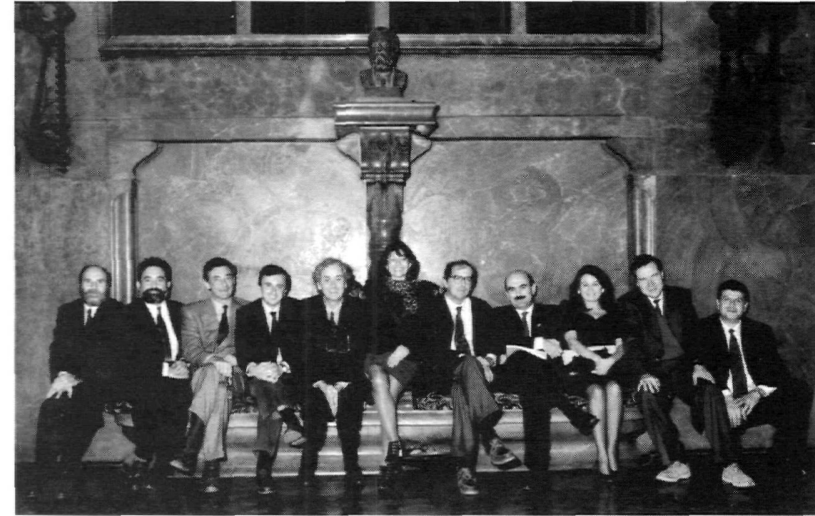

La asamblea fundacional de la Academia del Partal.

de un debate académico y una formación especializada son elementos imprescindibles para que la conservación del patrimonio deje de ser un aspecto olvidado y tome el papel que le corresponde en el conjunto de la práctica profesional" (18), dijo Muñoz Cosme en 1989, pero nada permite aventurar que ese momento se acerque.

En el panorama actual de la actuación en el patrimonio arquitectónico, con un horizonte abierto a la esperanza, pero tan lleno de nubarrones, quizá haya una circunstancia favorable: la nueva crisis económica que vivimos. Favorable, porque apaciguará definitivamente la fiebre de los años ochenta y, sobre todo, porque es posible que ahora, gracias a la nueva mentalidad a la que antes me refería, la escasez de recursos juegue a favor del fomento, junto a la

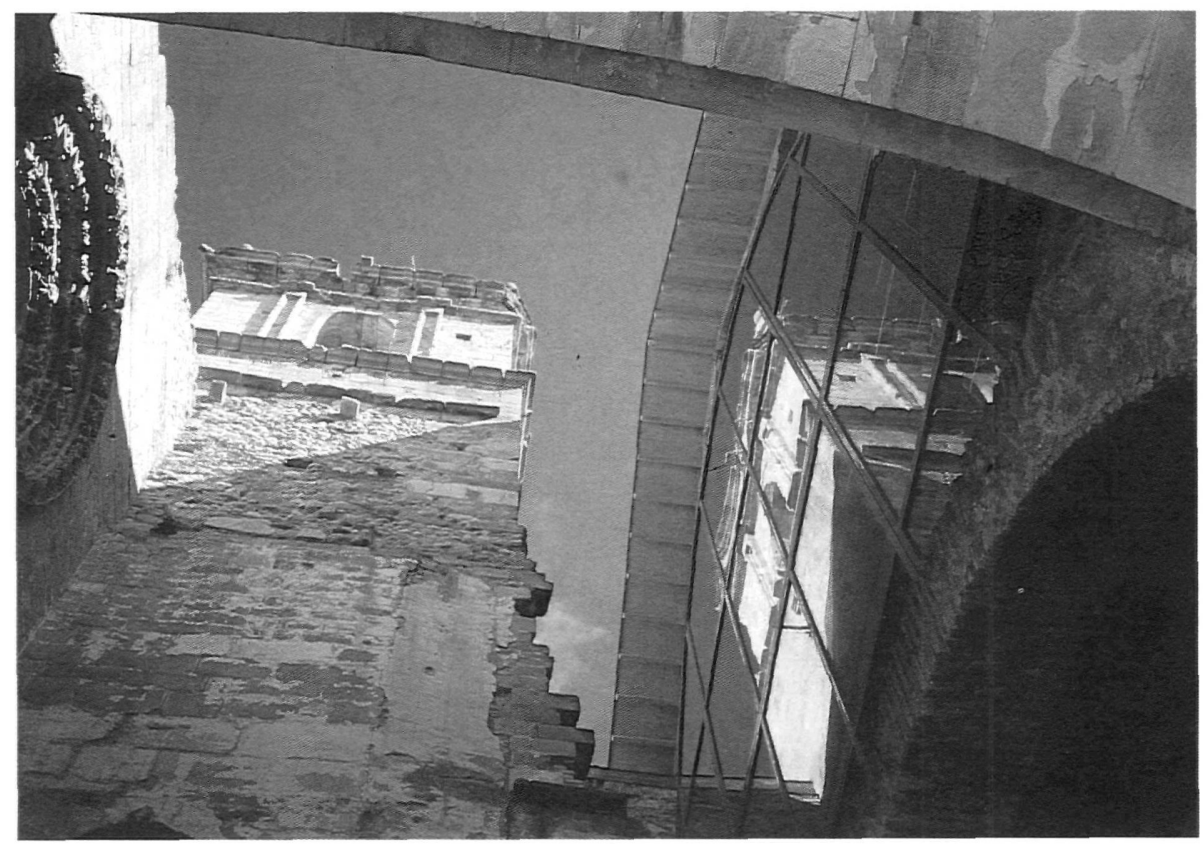

Iglesia del monasterio de Carracedo (León). 
cultura de la restauración, de una auténtica cultura de la conservación.

Una cultura de la conservación que han de asumir tanto los técnicos como la población en general. "Lo que a mí me parece más grave" -dice Javier Ramos en su excitante trabajo El final de la magia[es que] "hemos logrado, desgraciadamente, desvincular a los usuarios del natural proceso de conservación de sus edificios históricos, dando la -a mi juicio- falsa sensación de que ellos ni saben ni deben ocuparse de estas cosas. Creo" -concluye el arquitecto leonés- "que es el momento de cambiar de actitud ... Conservación y Restauración debieran formar parte de un proceso vital, desarrollado alrededor del edificio, como así ha sido para que éste tuviera lugar". (1"),

Conservación y restauración. Memoria y significado. Documento y arquitectura. Diálogo y especificidad. Diseño e investigación científica. Racionalidad y creatividad. Caras de una misma moneda que han de saber contemplar al mismo tiempo y valorar con la misma actitud quienes intervienen en la actuación en los monumentos, si pretenden que la disciplina, definitivamente, pase el rubicón.
(1.) Javier Rivera, "Restauraciones arquitectónicas y democracia en España", en $B A U$, n. 4, Vallactolid. 1990.

(2.) Antoni González Moreno-Navarro, "La restauración de monumentos a las puertas del siglo XXI". en Informes de la construcción, n. 413 Madrid, mayo-junio de 1991 .

13. José Gabriel Moya, "Así. no: A propósito de una intervención en Santa María la Real de Nájera. en Anuario del Departamento de Historia y Teoria del Arte". Universidąd Autónoma de Madrid. Vol. I., 1989.

(4.) Alfonso Munoz Cosme, La consenación del patrimonio arquitectónico español, ministerio de Cultura, Madrid, 1989.

(5.) Javier Rivera, "La restauración crítica y la problemática actual". en III Simposi sobre Restauració Monumental. "Quederns Cientifics i Técnics", n. 5, Diputación de Barcelona, Barcelona, noviembre de 1993.

(6.) Antón Capitel, Metamorfosis de monumentos y teorias de la restauración, Alianza Forma, Madrid. 1988; Alfonso Munozz Cosme, La conserlación... cit. supra, nota 4.: Jordi Ambrós. Lluís de Grassot, On Diseño, n. 102. Barcelona, 1989: Javier Rivera, "Restauración..., cit. supra. nota 1.

(7.) Javier Rivera, "La consolidación de la iglesia de la Clerecía de Salamanca y el debate sobre la restauración contemporínea en España", en A. Fernández Alba. Noticia de las obras de restauracion ." consolidación de la Real Clerecia de $S$. varcos en Salamanca. Instituro Espanol de Arquitectura. Madrid. junio de 1993.

(8.) Javier Rivera, "Restauraciones.... cit. supra. nota 1.

(9.) Alfonso Jiménez, "Notas para una tecria integrada de la restauración de monumentos". en Cuadernos de la comstrucción. Sevilla, mayo de 1982
(10.) Antoni Gonzále\%. "Patrinonio Arquitectónico: métodos de intervención" en Actas de la I conferencia Internacional sobre conseniación de Centros Históricos y. Parrimonio edificado Iberoamericano Alcalá de Henares. 26 al.31 de julio de 1993.

(11.) Antoni González, "A la recerca de la Restauració Objectiva". en Com $i$ per a qui restaurem. Objectius. Métodes i difusió de la restauració monumental. Memoria 1985-1989. Diputación de Barcelona, Barcelona, diciembre de 1990 .

(12.) Javier Rivera. "Lal restauración crítica... cit. supra, nota 5.

(13.) Primeros Encuentros Nacionales Eduardo Torroja. "Investigación Científica y Patrimonio Arquitectónico". León, 4.5 y 6 de marzo de 1993. Colegio Oficial de arquitectos de León. Junta de Castilla y León. CSIC (Instituto Torroja).

(14.) II Bienal de Arquitectura Española, 1991-1992, Santander y Comillas (Cantabria). julio-agosto de 1993. Ministerio de Obras Públicas y Transportes, Consejo Superior de los Colegios de Arquitectos de España, Liniversidad Internacional Menéndez y Pelayo.

(15.) Luis Fernández. Galiano (director). Anuario/Yearbook 1993 Arquitectura Espaniola Spanish Architecture, Avisa. Madrid, 1993.

(16.) Javier Rivera. "La restauración crítca... cit. supra, nota 6.

(17.) José Luis González Moreno-Navarro, El legado oculto de Vitrutio. Alianæa Forma. Madrid. 1993.

(18.) Alfonso Munoz Cosme, La consertación ... cit. supra. nota 4 .

(19.) Javier Ramos, "El final de la magia", en III Simposi sobre Restauració Momumenlal. "Quadern. Científics i Tècnics ", n. 5, Diputación de Barcelona. Barcelona. noviembre de 1993. 